\title{
A Family of Nonconforming Rectangular Elements for Strain Gradient Elasticity
}

\author{
Yulei Liao ${ }^{1}$ and Pingbing Ming ${ }^{2, *}$ \\ ${ }^{1}$ School of Mathematics, Renmin University of China, No. 59, Zhongguancun Street, \\ Beijing 100872, China \\ 2 LSEC, Institute of Computational Mathematics, AMSS Chinese Academy of \\ Sciences, No. 55, East Road Zhongguancun, Beijing 100190, China, and School of \\ Mathematical Sciences, University of Chinese Academy of Sciences, Beijing 100049, \\ China
}

Received 30 December 2018; Accepted (in revised version) 11 April 2019

\begin{abstract}
We propose a family of nonconforming rectangular elements for the linear strain gradient elastic model. Optimal error estimates uniformly with respect to the small material parameter have been proved. Numerical results confirm the theoretical prediction.
\end{abstract}

AMS subject classifications: $65 \mathrm{~N} 30,65 \mathrm{~N} 15,74 \mathrm{~K} 20$

Key words: Nonconforming finite elements, strain gradient elasticity, uniform error estimate.

\section{Introduction}

Strain gradient models play an important role in the characterization of the heterogeneity and the size effect of materials down to micro scale. Though it may date back to Cosserat brothers' classical work [12], numerical simulation of this model is rather recent [14,24, $27,30]$ because strain gradient models are usually quite complicate. In particular, they contain a couple of materials parameters and standard finite element approximations usually do not work for such model. Aifantis et al. [2,26] proposed a linear strain gradient elastic model that has only one material parameter. This simplified model successfully eliminated the strain singularity of the brittle crack tip field [13].

From a mathematical point of view, Aifantis' model is a singularly perturbed elliptic system of fourth order due to the appearance of the strain gradient. A natural choice for such type problems is $\mathrm{C}^{1}$ finite elements such as Argyris triangle [3], while the large number of local degrees of freedom and the high degree of polynomials used in the shape

${ }^{*}$ Corresponding author.

Emails: liaoyulei@ruc.edu.cn (Y. L. Liao), mpb@lsec.cc.ac.cn (P. B. Ming) 
functions are the main impediments for practical simulation. In [21], the authors proposed two nonconforming elements with 21 degrees of freedom and even simpler nonconforming elements have been recently constructed in [22]. The simplest one among them is the tensor product of the Morley's triangle [23] with a modification of the elastic strain energy. All elements converge uniformly with respect to the small material parameter.

In contrast to the triangle elements in [21,22], we consider the rectangular elements in the present work. The rectangular elements are equally powerful when they are combined with the isoparametric concept, which has been developed for Hermite elements in [25]. The famous Bogner-Fox-Schmit element (BFS) [6] has been exploited in [24,30] to approximate the strain gradient elastic model in two and three dimensions. The authors in [14] found that BFS outperforms several other elements in solving a nonlinear strain gradient elastic model. One drawback of BFS element is the large number of local degrees of freedom, the other is that the second order derivative appears in the definition of the degrees of freedom, which unfortunately brings in extra difficulty in dealing with boundary condition, because only the normal traction appears in the boundary condition of the strain gradient elastic model. To avoid such difficulty, we propose a family of rectangular element that is $\mathrm{H}^{2}$ nonconforming while $\mathrm{H}^{1}$ conforming. This means the finite element function is continuous, while the derivative is discontinuous across the element boundaries. Based on the discrete $\mathrm{H}^{2}$ inequality proved recently in [22], the tensor product of this element may be used to approximate the strain gradient elastic model. We prove that this element converges with optimal rate for both the smooth solution and the solution with a strong boundary layer. The latter is very common in the strain gradient elastic model.

The proposed element consists of the serendipity family of finite element [11] augmented with a special bubble space. This bubble space is a natural extension of the one appeared in [19]. Using the orthogonal properties of the Jacobi polynomials [28], we derive the explicit basis function associated with the bubble space, while the explicit representation of the corresponding bubble space in [19] is still unknown. Such basis functions of the bubble space could be exploited to construct the basis functions of the proposed element. It is worth pointing out that such family of rectangular element naturally yields a hierarchical nonconforming plate bending element, which obviously makes the nonconforming elements more competitive compared with the conforming element $[9,20,31]$ and the discontinuous Galerkin method; cf. [8]. It is worthwhile to mention that other orthogonal polynomials such as Legendre polynomials have been exploited to construct nonconforming element for plate bending element [17].

The structure of the paper is as follows. In Section 2, we introduce Aifantis' strain gradient model and the variational formulation. The finite element space is introduced in Section 3. Besides the structure of the element is clarified and the optimal approximation properties are proved for both smooth and nonsmooth functions. Error estimates are also proved in this part. The numerical results are reported in the last section. The explicit basis functions for the lowest order element and next to the lowest order element are 
included in the appendix.

Throughout this paper, the constant $C$ may differ from line to line, while it is independent of the mesh size $h$ and the material parameter $\iota$.

\section{Strain gradient elasticity model}

The space $L^{2}(\Omega)$ of the square-integrable functions defined on a bounded polygon $\Omega$ is equipped with the inner product $(\cdot, \cdot)$ and the norm $\|\cdot\|_{L^{2}(\Omega)}$. Let $H^{m}(\Omega)$ be the standard Sobolev space [1] with the norm $\|\cdot\|_{H^{m}(\Omega)}$ and the semi-norm $|\cdot|_{H^{m}(\Omega)}$. We may drop $\Omega$ in $\|\cdot\|_{H^{m}(\Omega)}$ when no confusion may occur. Define

$$
\begin{aligned}
& H_{0}^{1}(\Omega):=\left\{v \in H^{1}(\Omega) \mid v=0 \text { on } \partial \Omega\right\}, \\
& H_{0}^{2}(\Omega):=\left\{v \in H^{2}(\Omega) \mid v=0, \partial_{n} v=0 \text { on } \partial \Omega\right\},
\end{aligned}
$$

where $\partial_{n} v$ is the normal derivative of $v$. Equally, $\partial_{t} v$ denotes the tangential derivative of $v$.

For any vector-valued function $v$, its gradient is a matrix-valued function with components $(\nabla v)_{i j}=\partial v_{i} / \partial x_{j}$. The symmetric part of a gradient field is defined by $\epsilon(v)=$ $\left(\nabla v+[\nabla v]^{T}\right) / 2$. The divergence operator is defined as $\nabla \cdot v=\partial v_{1} / \partial x_{1}+\partial v_{2} / \partial x_{2}$. The Sobolev spaces $\left[H^{m}(\Omega)\right]^{2},\left[H_{0}^{m}(\Omega)\right]^{2}$ and $\left[L^{2}(\Omega)\right]^{2}$ of a vector field can be defined in a similar manner as their scalars counterpart. This rule equally applies to their inner products and their norms.

The strain gradient elastic model in $[2,26]$ is described by the following boundary value problem: For $u$ the displacement that solves

$$
\begin{cases}\left(\iota^{2} \Delta-I\right)(\mu \Delta u+(\lambda+\mu) \nabla \nabla \cdot u)=f & \text { in } \Omega, \\ u=\partial_{n} u=0 & \text { on } \partial \Omega,\end{cases}
$$

where $\lambda$ and $\mu$ are the Lamé constants and $\iota$ is the microscopic parameter such that $0<\iota \leq 1$. Here we assume that $0<\lambda<\infty$ and we do not consider the nearly incompressible materials, i.e., $\lambda \rightarrow \infty$, because the model may change dramatically for those materials [15]. Problem (2.1) may be rewritten into a variational problem: Find $u \in\left[H_{0}^{2}(\Omega)\right]^{2}$ such that

$$
a(u, v)=(f, v) \text { for all } v \in\left[H_{0}^{2}(\Omega)\right]^{2},
$$

where

$$
a(u, v):=(\mathbb{C} \epsilon(u), \epsilon(v))+\iota^{2}(\mathbb{D} \nabla \epsilon(u), \nabla \epsilon(v)),
$$

and the fourth-order tensor $\mathbb{C}$ and the sixth-order tensor $\mathbb{D}$ are defined as

$$
\mathbb{C}_{i j k l}=\lambda \delta_{i j} \delta_{k l}+2 \mu \delta_{i k} \delta_{j l} \quad \text { and } \quad \mathbb{D}_{i j k l m n}=\lambda \delta_{i l} \delta_{j k} \delta_{m n}+2 \mu \delta_{i l} \delta_{j m} \delta_{k n}
$$


respectively. Here $\delta_{i j}$ is the Kronecker delta function. The strain gradient $\nabla \epsilon(v)$ is a third order tensor that is defined by $(\nabla \epsilon(v))_{i j k}=\partial \epsilon_{j k} / \partial x_{i}$.

The variational problem (2.2) is well-posed if the bilinear form $a$ is coercive over $\left[H_{0}^{2}(\Omega)\right]^{2}$, which depends on the following inequality:

$$
\|\epsilon(v)\|_{L^{2}}^{2}+\|\nabla \epsilon(v)\|_{L^{2}}^{2} \geq \frac{1}{2}\|\nabla v\|_{H^{1}}^{2} \quad \text { for all } v \in\left[H_{0}^{2}(\Omega)\right]^{2} .
$$

This inequality was proved in [21, Theorem 1] by exploiting the community property of strain operator $\epsilon$ and the partial differential operator $\partial$. The regularity of the solution $u$ is given in the next lemma; see [22, Lemma 2.4] for a proof.

Lemma 2.1. There exists $C$ that may depend on $\Omega$ but independent of $\iota$ such that

$$
\begin{aligned}
& |u|_{H^{2}}+\iota|u|_{H^{3}} \leq C \iota^{-1 / 2}\|f\|_{L^{2}}, \\
& \left\|u-u_{0}\right\|_{H^{1}} \leq C \iota^{1 / 2}\|f\|_{L^{2},}
\end{aligned}
$$

where $u_{0} \in\left[H_{0}^{1}(\Omega)\right]^{2}$ satisfies

$$
\left(\mathbb{C} \epsilon\left(u_{0}\right), \epsilon(v)\right)=(f, v) \quad \text { for all } v \in\left[H_{0}^{1}(\Omega)\right]^{2} .
$$

Moreover, we have the estimate

$$
|u|_{H^{2}}|u|_{H^{3}} \leq C \iota^{-2}\|f\|_{L^{2}}^{2}
$$

\section{Rectangular element of any order}

In this part, we introduce a rectangular element of any order. Let $\mathcal{T}_{h}$ be the triangulation of $\Omega$ with rectangles $T:=\left(x_{T}-h_{x, T} / 2, x_{T}+h_{x, T} / 2\right) \times\left(y_{T}-h_{y, T} / 2, h_{y}+h_{y, T} / 2\right)$, where the mesh size $h=\max _{T \in \mathcal{T}_{h}} h_{T}$ with $h_{T}=\max \left(h_{x, T}, h_{y, T}\right)$. We assume that the mesh is shape regular in the sense that there exists a chunkiness parameter $\gamma$ such that $h_{x, T} / h_{y, T} \leq \gamma$. It is clear that $T$ is mapped into the unit square $K=I^{2}$ with $I=[-1,1]$ the unit interval. We shall work on $K$ instead of $T$ in what follows. Denote the set of all the edges in $\mathcal{T}_{h}$ as $\mathcal{E}_{h}$. The space of piecewise $\left[H^{m}\left(\Omega, \mathcal{T}_{h}\right)\right]^{2}$ vector fields is defined by

$$
\left[H^{m}\left(\Omega, \mathcal{T}_{h}\right)\right]^{2}:=\left\{v \in L^{2}(\Omega)|v|_{T} \in\left[H^{m}(T)\right]^{2} \quad \text { for all } T \in \mathcal{T}_{h}\right\},
$$

which is equipped with the norm

$$
\|v\|_{H_{h}^{k}}:=\|v\|_{L^{2}}+\sum_{k=1}^{m}\left\|\nabla_{h}^{k} v\right\|_{L^{2}}
$$

where

$$
\left\|\nabla_{h}^{k} v\right\|_{L^{2}}^{2}=\sum_{T \in \mathcal{T}_{h}}\left\|\nabla^{k} v\right\|_{L^{2}(T)}^{2}
$$

with $\left.\left(\nabla_{h}^{k} v\right)\right|_{T}=\left.\left(\nabla^{k} v\right)\right|_{T}$. 


\subsection{A new family of finite element}

Our construction is motivated by the element proposed in [19]. The serendipity finite element space $\mathcal{S}_{r}\left(I^{2}\right)[4]$ is defined by

$$
\mathcal{S}_{r}\left(I^{2}\right)=\mathcal{P}_{r}\left(I^{2}\right)+\operatorname{span}\left\{x^{r} y, x y^{r}\right\}
$$

where $\mathcal{P}_{r}\left(I^{2}\right)$ is the standard Lagrange finite element of degree $r$. The serendipity finite element space may be viewed as a reduction of the space $\mathcal{Q}_{r}\left(I^{2}\right)$, which is the tensor product Lagrange finite element space of degree $r \geq 2$. The degrees of freedom of $\mathcal{S}_{r}\left(I^{2}\right)$ are defined by

$$
v \mapsto \begin{cases}v(a) & \text { four vertices of } I^{2}, \\ \int_{e} v q & q \in \mathcal{P}_{r-2}(e) \quad \text { four edges } e, \\ \int_{I^{2}} v q & q \in \mathcal{P}_{r-4}\left(I^{2}\right) .\end{cases}
$$

We define the bubble function associated with $K$ as

$$
b_{K}=\frac{1}{16}\left(1-x^{2}\right)\left(1-y^{2}\right) \text {. }
$$

Let $e_{1}=[-1,1] \times\{-1\}, e_{2}=\{1\} \times[-1,1], e_{3}=[-1,1] \times\{1\}$ and $e_{4}=\{-1\} \times[-1,1]$. We define $\lambda_{1}=(1+y) / 2, \lambda_{2}=(1-x) / 2, \lambda_{3}=(1-y) / 2$ and $\lambda_{4}=(1+x) / 2$. It is clear that $\left.\lambda_{i}\right|_{e_{i}}=0$ for $i=1, \cdots, 4$. We also define the edge bubbles by $b_{e_{i}}=b_{K} / \lambda_{i}$.

The local finite element space

$$
P_{K}=\mathcal{S}_{r}\left(I^{2}\right)+b_{K} \sum_{e} b_{e} \Lambda_{e}\left(I^{2}\right)
$$

where

$$
\Lambda_{e}\left(I^{2}\right):=\left\{v \in \mathcal{P}_{r-2}\left(I^{2}\right) \mid \int_{I^{2}} b_{K} b_{e} v q \mathrm{~d} x=0 \quad \text { for all } q \in \mathcal{P}_{r-3}\left(I^{2}\right)\right\} .
$$

Now the set for the degrees of freedom $\Sigma_{K}$ consists of the degrees of freedom for $\mathcal{S}_{r}\left(I^{2}\right)$; cf. (3.1) and the moments

$$
v \mapsto \int_{e} \frac{\partial v}{\partial n} q, \quad q \in \mathcal{P}_{r-2}(e)
$$

The number of the degrees of freedom is

$$
\# \Sigma_{K}=4+4(r-1)+\left(\begin{array}{c}
r-2 \\
2
\end{array}\right)+4(r-1)=\left(\begin{array}{c}
r+2 \\
2
\end{array}\right)+2+4(r-1)
$$

where the binomial $\left(\begin{array}{c}r \\ 2\end{array}\right):=r(r-1) / 2$.

We are ready to prove the unisolvence of the above finite element. 
Lemma 3.1. The set $\left(K, P_{K}, \Sigma_{K}\right)$ is unisolvent.

Proof. We only need to show that if all the degrees of freedom vanish, $v$ vanishes. Note that the first set of degrees of freedom is zero means that $v=b_{K} \sum_{e} b_{e} q_{e}$ with $q_{e} \in \Lambda_{e}\left(I^{2}\right)$. It is clear that for any edge $E \subset I^{2}$, we have

$$
\left.\frac{\partial v}{\partial n}\right|_{E}=\left.\left.\frac{\partial b_{K}}{\partial n}\right|_{E}\left(\sum_{e} b_{e} q_{e}\right)\right|_{E}+b_{K}\left|E\left(\frac{\partial}{\partial n} \sum_{e} b_{e} q_{e}\right)\right|_{E}=-\left.\frac{1}{2}\left(b_{E}^{2} q_{E}\right)\right|_{E} .
$$

Note that

$$
\int_{E} \frac{\partial v}{\partial n} q=0 \quad \text { for all } q \in \mathcal{P}_{r-2}(E)
$$

Taking $q=q_{E}$ in the above identity, we obtain

$$
\int_{E} b_{E}^{2} q_{E}^{2}=0
$$

which immediately implies that $\left.q_{E}\right|_{E}=0$. Therefore, we write $q_{E}=\lambda_{E} p_{E}$ with $p_{E} \in \mathcal{P}_{r-3}\left(I^{2}\right)$. By the definition of $q_{E}$, we have, for any $q \in \mathcal{P}_{r-3}\left(I^{2}\right)$,

$$
0=\int_{I^{2}} b_{K} b_{E} q_{E} q=\int_{I^{2}} b_{K} b_{E} \lambda_{E} p_{E} q=\int_{I^{2}} b_{K}^{2} p_{E} q .
$$

Taking $q=p_{E} \in \mathcal{P}_{r-3}\left(I^{2}\right)$ in the above identity, we obtain $p_{E} \equiv 0$ in $I^{2}$. So does $q_{E}$ and $v$. This proves the unisolvency.

Example 3.1. The enriched bubble space for the lowest order case $r=2$ is

$$
b_{K} \operatorname{span}\left\{b_{e_{1}}, b_{e_{2}}, b_{e_{3}}, b_{e_{4}}\right\} \text {. }
$$

The basis functions associated with the degrees of freedom $\int_{e_{i}} \partial v / \partial n$ are

$$
-30 b_{K} b_{e_{1}}, \quad-30 b_{K} b_{e_{2}}, \quad 30 b_{K} b_{e_{3}}, \quad 30 b_{K} b_{e_{4}} .
$$

Remark 3.1. A drawback of this approach is that the degree of the enriched bubble space is slightly high. The authors in [10] proposed the following choices for the bubble spaces:

$$
\begin{cases}b_{K} \operatorname{span}\left\{x, x^{2}, y, y^{2}\right\}, & r=2 \\ b_{K} \operatorname{span}\left\{x, x^{2} y, x^{3} y, x^{4}, y, y^{2} x, y^{3} x, y^{4}\right\}, & r=3\end{cases}
$$

Unfortunately, it does not seem easy to extend such Ansatz for arbitrary high order. 
Next we clarify the structure of the bubble space. To this end, we need the following facts about the Jacobi polynomials [28]. For any $\alpha, \beta>-1$ and nonnegative integers $n, m$, there holds

$$
\int_{-1}^{1}(1-x)^{\alpha}(1+x)^{\beta} P_{n}^{(\alpha, \beta)}(x) P_{m}^{(\alpha, \beta)}(x) \mathrm{d} x=h_{n}^{(\alpha, \beta)} \delta_{n m}
$$

where

$$
h_{n}^{(\alpha, \beta)}=\int_{-1}^{1}(1-x)^{\alpha}(1+x)^{\beta}\left[P_{n}^{(\alpha, \beta)}(x)\right]^{2} \mathrm{~d} x .
$$

By [28, Eq. (4.3.3)], we may write

$$
h_{n}^{(\alpha, \beta)}=\frac{2^{\alpha+\beta+1}}{2 n+\alpha+\beta+1} \frac{\Gamma(n+\alpha+1) \Gamma(n+\beta+1)}{\Gamma(n+\alpha+\beta+1) \Gamma(n+1)},
$$

where $\Gamma$ is the Gamma function.

One of the explicit form for $P_{n}^{(\alpha, \beta)}$ is

$$
(1-x)^{\alpha}(1+x)^{\beta} P_{n}^{(\alpha, \beta)}(x)=\frac{(-1)^{n}}{2^{n} n !} \frac{\mathrm{d}^{n}}{\mathrm{~d} x^{n}}\left((1-x)^{n+\alpha}(1+x)^{n+\beta}\right) .
$$

In particular,

$$
P_{0}^{(\alpha, \beta)}(x)=1, \quad P_{1}^{(\alpha, \beta)}(x)=\frac{1}{2}(\alpha+\beta+2) x+\frac{1}{2}(\alpha-\beta) .
$$

Lemma 3.2. The bubble spaces take the following form:

$$
\left\{\begin{array}{l}
\Lambda_{e_{1}}\left(I^{2}\right)=\operatorname{span}\left\{P_{n}^{(2,2)}(x) P_{m}^{(2,1)}(y)\right\}, \\
\Lambda_{e_{2}}\left(I^{2}\right)=\operatorname{span}\left\{P_{n}^{(1,2)}(x) P_{m}^{(2,2)}(y)\right\}, \\
\Lambda_{e_{3}}\left(I^{2}\right)=\operatorname{span}\left\{P_{n}^{(2,2)}(x) P_{m}^{(1,2)}(y)\right\}, \\
\Lambda_{e_{4}}\left(I^{2}\right)=\operatorname{span}\left\{P_{n}^{(2,1)}(x) P_{m}^{(2,2)}(y)\right\},
\end{array}\right.
$$

where $n, m$ are nonegative integers and $n+m=r-2$.

Proof. We only prove the first identity in (3.6). Others may be proceeded similarly.

Any $p \in \mathcal{P}_{r-2}\left(I^{2}\right)$ may be expanded into

$$
p(x, y)=\sum_{0 \leq n+m \leq r-2} a_{n m} P_{n}^{(2,2)}(x) P_{m}^{(2,1)}(y)
$$

for certain parameters $a_{n m}$. Using the above representation (3.7), we may write the constraint in the definition of bubble space (3.2) $\Lambda_{e_{1}}$ as

$$
\int_{I^{2}}\left(1-x^{2}\right)^{2}\left(1-y^{2}\right)(1-y) \sum_{n, m} a_{n m} P_{n}^{(2,2)}(x) P_{m}^{(2,1)}(y) q(x, y) \mathrm{d} x \mathrm{~d} y=0
$$


for all $q \in \mathcal{P}_{r-3}\left(I^{2}\right)$. We substitute $q=P_{i}^{(2,2)}(x) P_{j}^{(2,1)}(y)$ with $0 \leq i+j \leq r-3$ into the above equation. Using the orthogonal relation (3.3) for the Jacobi polynomials, we may obtain

$$
a_{i j}=0 \text { for all } 0 \leq i+j \leq r-3 .
$$

This immediately implies (3.6).

Motivated by Lemma 3.2, we change the definition of the degrees of freedom associated with the bubble space to

$$
\begin{aligned}
& \int_{e_{i}} \frac{\partial v}{\partial n} P_{m}^{(2,2)}(x) \mathrm{d} x, \quad i=1,3 \text { and } m=0, \cdots, r-2, \\
& \int_{e_{i}} \frac{\partial v}{\partial n} P_{m}^{(2,2)}(y) \mathrm{d} y, \quad i=2,4 \text { and } m=0, \cdots, r-2 .
\end{aligned}
$$

This may yield a compact form of the basis functions for the bubble space.

Lemma 3.3. The basis functions for the bubble spaces are: for $n, m$ nonnegative integers with $n+m=r-2$,

$$
\begin{aligned}
a_{n m}^{(1)} b_{K} b_{e_{1}} P_{n}^{(2,2)}(x) P_{m}^{(2,1)}(y), & a_{n m}^{(1)}=-\frac{32}{h_{n}^{(2,2)} P_{m}^{(2,1)}(-1)^{\prime}} \\
a_{n m}^{(2)} b_{K} b_{e_{2}} P_{n}^{(1,2)}(x) P_{m}^{(2,2)}(y), & a_{n m}^{(2)}=-\frac{32}{h_{m}^{(2,2)} P_{n}^{(1,2)}(1)}, \\
a_{n m}^{(3)} b_{K} b_{e_{3}} P_{n}^{(2,2)}(x) P_{m}^{(1,2)}(y), & a_{n m}^{(3)}=\frac{32}{h_{n}^{(2,2)} P_{m}^{(1,2)}(1)}, \\
a_{n m}^{(4)} b_{K} b_{e_{4}} P_{n}^{(2,1)}(x) P_{m}^{(2,2)}(y), & a_{n m}^{(4)}=\frac{32}{h_{m}^{(2,2)} P_{n}^{(2,1)}(-1)} .
\end{aligned}
$$

Here

$$
\begin{aligned}
& a_{n m}^{(1)}=\frac{(-1)^{m+1}}{m+1} \frac{(n+3)(n+4)(2 n+5)}{(n+1)(n+2)}, \\
& a_{n m}^{(2)}=(-1)^{n} a_{m n}^{(1)}, \quad a_{n m}^{(3)}=(-1)^{m+1} a_{n m}^{(1)}, \quad a_{n m}^{(4)}=-a_{m n}^{(1)} .
\end{aligned}
$$

Proof. We only prove (3.8a). Others may be proceeded similarly.

Firstly, a direct calculation gives

$$
\left.\left(\frac{\partial}{\partial n}\left(b_{K} b_{e_{1}} P_{n}^{(2,2)}(x) P_{m}^{(2,1)}(y)\right)\right)\right|_{e_{1}}=-\left.\frac{1}{2}\left[b_{e_{1}}^{2}\right]\right|_{e_{1}} P_{n}^{(2,2)}(x) P_{m}^{(2,1)}(-1) .
$$


For $i=0, \cdots, r-2$, we obtain

$$
\begin{aligned}
& \int_{e_{1}} P_{i}^{(2,2)}(x) \frac{\partial}{\partial n}\left(b_{K} b_{e_{1}} P_{n}^{2,2}(x) P_{m}^{(2,1)}(y)\right) \mathrm{d} x \\
= & -\frac{1}{32} P_{m}^{(2,1)}(-1) \int_{-1}^{1}(1-x)^{2}(1+x)^{2} P_{n}^{(2,2)}(x) P_{i}^{(2,2)}(x) \mathrm{d} x \\
= & -\frac{1}{32} P_{m}^{(2,1)}(-1) h_{i}^{(2,2)} \delta_{i n} .
\end{aligned}
$$

This gives (3.8a).

Next, using [28, Eq. (4.1.1)], we obtain

$$
P_{n}^{(1,2)}(1)=\left(\begin{array}{c}
n+1 \\
n
\end{array}\right)=n+1
$$

Using [28, Eq. (4.1.3)], we obtain

$$
P_{n}^{(2,1)}(-1)=(-1)^{n}\left(\begin{array}{c}
n+1 \\
n
\end{array}\right)=(-1)^{n}(n+1),
$$

and using (3.4), we obtain

$$
h_{n}^{(2,2)}=\frac{32(n+1)(n+2)}{(n+3)(n+4)(2 n+5)}
$$

Substituting the above three formulae into (3.8a), we obtain the expression of $a_{n m}^{(1)}$.

Remark 3.2. The bubble space may be extended to three dimension, which reads as $b_{K} \sum_{f} b_{f} \Lambda_{f}\left(I^{3}\right)$ with

$$
\Lambda_{f}\left(I^{3}\right):=\left\{v \in \mathcal{P}_{r-2}\left(I^{3}\right) \mid \int_{I^{3}} b_{K} b_{f} v q=0 \quad \text { for all } q \in \mathcal{P}_{r-3}\left(I^{3}\right)\right\} .
$$

The associated degrees of freedom are

$$
\int_{f} \frac{\partial v}{\partial n} q, \quad q \in \mathcal{P}_{r-2}(f)
$$

Here the summation runs for all faces $f$. The bubble function

$$
b_{K}=\frac{1}{64}\left(1-x^{2}\right)\left(1-y^{2}\right)\left(1-z^{2}\right)
$$

and the face bubble $b_{f}=b_{K} / \lambda_{f}$ with $\left.\lambda_{f}\right|_{f} \equiv 0$. 
Given $\left(K, P_{K}, \Sigma_{K}\right)$, we may define the finite element triple $\left(T, W(T), \Sigma_{T}\right)$. The corresponding finite element space is defined by

$$
X_{h}^{0}:=\left\{v \in H_{0}^{1}(\Omega)|v|_{T} \in W(T)\right\},
$$

and we may define the approximation space for the strain gradient elastic model as $V_{h}^{0}=$ $\left[X_{h}^{0}\right]^{2}$. The approximation problem reads as: Find $u_{h} \in V_{h}^{0}$ such that

$$
a_{h}\left(u_{h}, v\right)=(f, v) \text { for all } v \in V_{h}^{0},
$$

where the bilinear form $a_{h}$ is defined for any $v, w \in V_{h}^{0}$ as

$$
a_{h}(v, w):=(\mathbb{C} \epsilon(v), \epsilon(w))+\iota^{2}\left(\mathbb{D} \nabla_{h} \epsilon(v), \nabla_{h} \epsilon(w)\right)
$$

with

$$
\left(\mathbb{D} \nabla_{h} \epsilon(v), \nabla_{h} \epsilon(w)\right):=\sum_{T \in \mathcal{T}_{h}} \int_{T} \mathbb{D} \nabla \epsilon(v) \nabla \epsilon(w) \mathrm{d} x
$$

The energy norm is defined as $\|v \mid\|_{\iota, h}=\|\nabla v\|_{L^{2}}+\iota\left\|\nabla_{h}^{2} v\right\|_{L^{2}}$. This is indeed a norm because $\|v \mid\|_{\iota, h}$ is equivalent to $\|v\|_{H^{1}}+\left\|\nabla_{h}^{2} v\right\|_{L^{2}}$ for any fixed $\iota$ by virtue of Poincaré inequality for $v \in\left[H_{0}^{1}(\Omega)\right]^{2}$.

\subsection{Approximation properties of the finite elements}

Using the degrees of freedom given in the last section, we define a local interpolation operator $\Pi_{T}: H^{2}(T) \longmapsto W(T)$ as: For all vertices $a$ and all edges $e$,

$$
\begin{cases}\Pi_{T} v(a)=v(a) & \\ \int_{e} \Pi_{T} v(x) p(x) \mathrm{d} \sigma(x)=\int_{e} v(x) p(x) \mathrm{d} \sigma(x) & \text { for all } p \in \mathcal{P}_{r-2}(e) \\ \int_{T} \Pi_{T} v(x) q(x) \mathrm{d} x=\int_{T} v(x) q(x) \mathrm{d} x & \text { for all } q \in \mathcal{P}_{r-4}(T) \\ \int_{e} \frac{\partial \Pi_{T} v}{\partial n}(x) p(x) \mathrm{d} x=\int_{e} \frac{\partial v}{\partial n}(x) p(x) \mathrm{d} x & \text { for all } q \in \mathcal{P}_{r-2}(e)\end{cases}
$$

To prove the interpolation error estimate, we firstly show that $\Pi_{T}$ is $\mathcal{S}_{r}(T)$ invariant.

\section{Lemma 3.4.}

$$
\Pi_{T} v=v \text { for all } v \in \mathcal{S}_{r}(T) .
$$

The above lemma and the proof below is adapted from [21, Lemma 3]. We give the details of the proof for the convenience of the readers. 
Proof. Denote $M=\operatorname{dim} \mathcal{S}_{r}(T)$ and $N=\operatorname{dim} b(T)$, i.e., $M=4 r+(r-2)(r-1) / 2$ and $N=$ $4 r-4$. Let $\left\{\omega_{i}\right\}_{i=1}^{M}$ be the basis functions for $\mathcal{S}_{r}(T)$ associated with degrees of freedom $\left\{\phi_{i}^{(n)}\right\}_{i=1}^{M}$ and let $\left\{\psi_{i}\right\}_{i=1}^{N}$ be the basis functions for the bubble space $b(T)$ associated with the degrees of freedom $\left\{\phi_{i}^{(m)}\right\}_{i=1}^{N}$. For $i=1, \cdots, M$, define

$$
\varphi_{i}=\omega_{i}-\sum_{j=1}^{N} \phi_{j}^{(m)}\left(\omega_{i}\right) \psi_{j} .
$$

We claim that

$$
W(T)=\operatorname{span}\left\{\varphi_{1}, \cdots, \varphi_{M}, \psi_{1}, \cdots, \psi_{N}\right\} .
$$

Note that $\phi_{i}^{(n)}\left(\psi_{j}\right) \equiv 0$ for any $i=1, \cdots, M$ and $j=1, \cdots, N$, because $\psi_{j}$ vanishes on $\partial T$. Thus we obtain that $\left\{\psi_{i}\right\}_{i=1}^{N}$ are the basis functions for $W(T)$ associated with the degrees of freedom $\left\{\phi_{j}^{(m)}\right\}_{j=1}^{N}$. For any $\varphi_{i}$, there holds

$$
\begin{aligned}
& \phi_{j}^{(n)}\left(\varphi_{i}\right)=\phi_{j}^{(n)}\left(\omega_{i}\right)-\sum_{k=1}^{N} \phi_{k}^{(m)}\left(\omega_{i}\right) \phi_{j}^{(n)}\left(\psi_{k}\right)=\phi_{j}^{(n)}\left(\omega_{i}\right)=\delta_{i j} \\
& \phi_{j}^{(m)}\left(\varphi_{i}\right)=\phi_{j}^{(m)}\left(\omega_{i}\right)-\sum_{k=1}^{N} \phi_{k}^{(m)}\left(\omega_{i}\right) \phi_{j}^{(m)}\left(\psi_{k}\right)=\phi_{j}^{(m)}\left(\omega_{i}\right)-\sum_{k=1}^{N} \phi_{k}^{(m)}\left(\omega_{i}\right) \delta_{k j}=0 .
\end{aligned}
$$

This verifies (3.11).

Next we prove the interpolation operator is locally $\mathcal{S}_{r}$-invariant. In fact, for any $v \in \mathcal{S}_{r}(T)$, we have the representation

$$
v=\sum_{i=1}^{M} \phi_{i}^{(n)}(v) \omega_{i} .
$$

By definition,

$$
\begin{aligned}
\Pi_{T} v & =\sum_{i=1}^{M} \phi_{i}^{(n)}(v) \varphi_{i}+\sum_{j=1}^{N} \phi_{j}^{(m)}(v) \psi_{j} \\
& =\sum_{i=1}^{M} \phi_{i}^{(n)}(v) \omega_{i}-\sum_{j=1}^{N} \sum_{i=1}^{M} \phi_{i}^{(n)}(v) \phi_{j}^{(m)}\left(\omega_{i}\right) \psi_{j}+\sum_{j=1}^{N} \phi_{j}^{(m)}(v) \psi_{j} \\
& =v+\sum_{j=1}^{N}\left(\phi_{j}^{(m)}(v)-\sum_{i=1}^{M} \phi_{i}^{(n)}(v) \phi_{j}^{(m)}\left(\omega_{i}\right)\right) \psi_{j} \\
& =v,
\end{aligned}
$$

where we have used the fact that

$$
\phi_{j}^{(m)}(v)=\sum_{i=1}^{M} \phi_{i}^{(n)}(v) \phi_{j}^{(m)}\left(\omega_{i}\right)
$$

in the last step. 
The claim (3.11) gives a constructive way for deriving the basis functions of the proposed element. To this end, one may use the basis functions derived in [16] for the serendipity family of finite element and the basis functions given in Lemma 3.3 for the bubble space. We shall construct the basis functions for the element with $r=2,3$ in the Appendix.

We shall use the following versions of the trace inequalities.

Lemma 3.5. For any Lipschitz domain $D$, there exists $C$ depending on $D$ such that

$$
\|v\|_{L^{2}(\partial D)} \leq C\|v\|_{L^{2}(D)}^{1 / 2}\|v\|_{H^{1}(D)}^{1 / 2} .
$$

For any element $T \in \mathcal{T}_{h}$, there exists $C$ independent of $h_{T}$, but depends on $\gamma$ such that

$$
\|v\|_{L^{2}(\partial T)} \leq C\left(h_{T}^{-1 / 2}\|v\|_{L^{2}(T)}+\|v\|_{L^{2}(T)}^{1 / 2}\|\nabla v\|_{L^{2}(T)}^{1 / 2}\right) .
$$

The multiplicative type trace inequality (3.12) may be found in [18], while (3.13) is a direct consequence of (3.12).

The main result of this section is the following interpolate estimate.

Lemma 3.6. There exists $C$ that depends on the chunkiness parameter $\gamma$ such that

$$
\begin{aligned}
& \sum_{j=0}^{2} h_{T}^{j}\left\|\nabla^{j}\left(u-\Pi_{T} u\right)\right\|_{L^{2}(T)} \leq C h_{T}^{m}|u|_{H^{m}(T)}, \quad m=2, \cdots, r+1, \\
& \left\|\nabla\left(v-\Pi_{T} v\right)\right\|_{L^{2}(T)} \leq C h_{T}^{1 / 2}\|\nabla v\|_{L^{2}(T)}^{1 / 2}\left(\left\|\partial_{x}^{2} v\right\|_{L^{2}(T)}+\left\|\partial_{y}^{2} v\right\|_{L^{2}(T)}\right)^{1 / 2} .
\end{aligned}
$$

More convenient but less sharp form of $(3.14 \mathrm{~b})$ is

$$
\left\|\nabla\left(v-\Pi_{T} v\right)\right\|_{L^{2}(T)} \leq C h_{T}^{1 / 2}\|\nabla v\|_{L^{2}(T)}^{1 / 2}\left\|\nabla^{2} v\right\|_{L^{2}(T)}^{1 / 2} .
$$

Proof. The first interpolation error estimate (3.14a) is a direct consequence of the $\mathcal{S}_{r}(T)$-invariance of the interpolation operator proved in Lemma 3.4.

The second interpolation estimate (3.14b) is much more involved, which is crucial for deriving the uniform error estimate. The proof here essentially follows that in [22, Lemma 4.1] with a minor modification.

Using a standard scaling argument, we obtain

$$
\begin{aligned}
\left\|\nabla\left(v-\Pi_{T} v\right)\right\|_{L^{2}(T)} & \leq C\left\|\widehat{\nabla}\left(\widehat{v}-\widehat{\Pi}_{K} \widehat{v}\right)\right\|_{L^{2}(K)} \\
& \leq C\left(\|\widehat{\nabla} \widehat{v}\|_{L^{2}(K)}+\|\widehat{v}\|_{L^{\infty}(K)}+\|\widehat{\nabla} \widehat{v}\|_{L^{2}(\partial K)}\right) .
\end{aligned}
$$

The left-hand side is invariant if we replace $v$ by $v-c$ for any constant $c$. Therefore, we may rewrite the above inequality into

$$
\left\|\widehat{\nabla}\left(\widehat{v}-\widehat{\Pi}_{K} \widehat{v}\right)\right\|_{L^{2}(K)} \leq C\left(\|\widehat{\nabla} \widehat{v}\|_{L^{2}(K)}+\|\widehat{v}-c\|_{L^{\infty}(K)}+\|\widehat{\nabla} \widehat{v}\|_{L^{2}(\partial K)}\right) .
$$


By Sobolev imbedding theorem $W^{1, p}(K) \hookrightarrow L^{\infty}(K)$ [1] for any $p>2$, we obtain that for any $2<p<4$, there holds

$$
\|\widehat{v}-c\|_{L^{\infty}(K)} \leq C\left(\|\widehat{v}-c\|_{L^{p}(K)}+\|\widehat{\nabla} \widehat{v}\|_{L^{p}(K)}\right) \leq C\|\widehat{\nabla} \widehat{v}\|_{L^{p}(K)},
$$

where we have used the Poincaré inequality over $K$ in the last step.

Next, by interpolation inequality and the Sobolev imbedding inequality $H^{1}(K) \hookrightarrow$ $L^{q}(K)$ with $q=2 p /(4-p)$, there holds

$$
\|\widehat{\nabla} \widehat{v}\|_{L^{p}(K)} \leq\|\widehat{\nabla} \widehat{v}\|_{L^{2}(K)}^{1 / 2}\|\widehat{\nabla} \widehat{v}\|_{L^{q}(K)}^{1 / 2} \leq C\|\widehat{\nabla} \widehat{v}\|_{L^{2}(K)}^{1 / 2}\|\widehat{\nabla} \widehat{v}\|_{H^{1}(K)}^{1 / 2}
$$

Combining the above three inequalities and using the multiplicative trace inequality (3.12) to bound $\|\widehat{\nabla} \widehat{v}\|_{L^{2}(\partial K)}$, we obtain

$$
\left\|\widehat{\nabla}\left(\widehat{v}-\widehat{\Pi}_{K} \widehat{v}\right)\right\|_{L^{2}(K)} \leq C\|\widehat{\nabla} \widehat{v}\|_{L^{2}(K)}^{1 / 2}\|\widehat{\nabla} \widehat{v}\|_{H^{1}(K)}^{1 / 2} .
$$

Note $\widehat{\Pi}$ is affine-invariant for the bilinear element space $Q_{1}(K)$, then we have

$$
\left\|\widehat{\nabla}\left(\widehat{v}-\widehat{\Pi}_{K} \widehat{v}\right)\right\|_{L^{2}(K)} \leq C \inf _{q \in \mathbb{Q}_{1}(K)}\|\widehat{\nabla}(\widehat{v}-q)\|_{L^{2}(K)}^{1 / 2}\|\widehat{\nabla}(\widehat{v}-q)\|_{H^{1}(K)}^{1 / 2} .
$$

We take $q$ as the Galerkin projection of $\widehat{v}$ in the sense that $q \in \mathbb{Q}_{1}(K)$ satisfying

$$
\int_{K} \widehat{\nabla}(\widehat{v}-q) \widehat{\nabla} \widehat{w}=0 \quad \text { for all } \widehat{w} \in \mathbb{Q}_{1}(K) .
$$

By error estimate for the Galerkin projection [7], we have

$$
\begin{aligned}
& \|\widehat{\nabla}(\widehat{v}-q)\|_{L^{2}(K)} \leq\|\widehat{\nabla} \widehat{v}\|_{L^{2}(K)}, \\
& \|\widehat{\nabla}(\widehat{v}-q)\|_{H^{1}(K)} \leq C\left(\left\|\widehat{\partial}_{\widehat{x}}^{2} \widehat{v}\right\|_{L^{2}(K)}+\left\|\widehat{\partial}_{\widehat{y}}^{2} \widehat{v}\right\|_{L^{2}(K)}\right) .
\end{aligned}
$$

Combining the above three estimates, we obtain

$$
\left\|\widehat{\nabla}\left(\widehat{v}-\widehat{\Pi}_{K} \widehat{v}\right)\right\|_{L^{2}(K)} \leq C\|\widehat{\nabla} \widehat{v}\|_{L^{2}(K)}^{1 / 2}\left(\left\|\widehat{\partial}_{\widehat{x}}^{2} \widehat{v}\right\|_{L^{2}(K)}+\left\|\widehat{\partial}_{\widehat{y}}^{2} \widehat{v}\right\|_{L^{2}(K)}\right)^{1 / 2} .
$$

A standard scaling argument yields (3.14b).

A direct consequence of Lemma 3.6 is the following approximation results with respect to the energy norm.

Lemma 3.7. There exists $C$ that depends on the chunkiness parameter $\gamma$ such that

$$
\begin{aligned}
& \inf _{v \in V_{h}^{0}}|| u-v|\||_{L, h} \leq C h^{r-1}\left(h|u|_{H^{r+1}}+\iota|u|_{H^{r+2}}\right), \\
& \inf _{v \in V_{h}^{0}}\|u-v \mid\|\left\|_{L, h} \leq C h^{1 / 2}\right\| f \|_{L^{2}} .
\end{aligned}
$$


Proof. Let $v=\Pi_{h} u=\left(\Pi_{h} u_{1}, \Pi_{h} u_{2}\right)$ with $\left(\Pi_{h}\right)_{T}=\Pi_{T}$. The interpolation estimate (3.16a) follows from (3.14a).

Using (3.15), we obtain

$$
\begin{aligned}
\left\|\nabla\left(u-\Pi_{h} u\right)\right\|_{L^{2}} & \leq\left\|\nabla\left(I-\Pi_{h}\right)\left(u-u_{0}\right)\right\|_{L^{2}}+\left\|\nabla\left(u_{0}-\Pi_{h} u_{0}\right)\right\|_{L^{2}} \\
& \leq C h^{1 / 2}\left\|\nabla\left(u-u_{0}\right)\right\|_{L^{2}}^{1 / 2}\left\|\nabla^{2}\left(u-u_{0}\right)\right\|_{L^{2}}^{1 / 2}+C h\left\|\nabla^{2} u_{0}\right\|_{L^{2}} .
\end{aligned}
$$

Substituting $(2.4 \mathrm{~b})$ and (3.16b) into the above inequality, we obtain

$$
\left\|\nabla\left(u-\Pi_{h} u\right)\right\|_{L^{2}} \leq C h^{1 / 2}\|f\|_{L^{2}} .
$$

Using (3.14a) with $j=m=2$, we have

$$
\left\|\nabla^{2}\left(u-\Pi_{h} u\right)\right\|_{L^{2}} \leq C\left\|\nabla^{2} u\right\|_{L^{2}}
$$

Using (3.14a) with $j=2$ and $m=3$, we have

$$
\left\|\nabla^{2}\left(u-\Pi_{h} u\right)\right\|_{L^{2}} \leq C h\left\|\nabla^{3} u\right\|_{L^{2}} .
$$

Interpolating between the above two inequalities, we obtain

$$
\left\|\nabla^{2}\left(u-\Pi_{h} u\right)\right\|_{L^{2}} \leq C h^{1 / 2}\left\|\nabla^{2} u\right\|_{L^{2}}^{1 / 2}\left\|\nabla^{3} u\right\|_{L^{2}}^{1 / 2} .
$$

Using the regularity estimate (2.6), we obtain

$$
\iota\left\|\nabla^{2}\left(u-\Pi_{h} u\right)\right\|_{L^{2}} \leq C h^{1 / 2}\|f\|_{L^{2}}
$$

which together with (3.17) implies (3.16b).

The following coercivity inequality with respect to the energy norm is crucial for wellposedness and the error estimate of the proposed method. The proof is adapted from [22, Theorem 2.1].

Lemma 3.8. There holds

$$
a_{h}(v, v) \geq(2-\sqrt{2}) \mu \mid\|v\|_{\iota, h}^{2} \quad \text { for all } v \in V_{h}^{0} .
$$

Proof. For any $v \in V_{h}^{0}$,

$$
a_{h}(v, v) \geq 2 \mu\left(\|\epsilon(v)\|_{L^{2}}^{2}+\iota^{2}\left\|\nabla_{h} \epsilon(v)\right\|_{L^{2}}^{2}\right) .
$$

For any $v \in V_{h}^{0} \subset\left[H_{0}^{1}(\Omega)\right]^{2}$, we have the first Korn's inequality

$$
2\|\epsilon(v)\|_{L^{2}}^{2} \geq\|\nabla v\|_{L^{2}}^{2} .
$$


A direct calculation gives

$$
\begin{aligned}
|\nabla \epsilon(v)|^{2}=\left|\frac{\partial^{2} v_{1}}{\partial x_{1}^{2}}\right|^{2} & +\left|\frac{\partial^{2} v_{1}}{\partial x_{1} \partial x_{2}}\right|^{2}+\left|\frac{\partial^{2} v_{2}}{\partial x_{1} \partial x_{2}}\right|^{2}+\left|\frac{\partial^{2} v_{2}}{\partial x_{2}^{2}}\right|^{2} \\
& +\frac{1}{2}\left|\frac{\partial^{2} v_{1}}{\partial x_{1} \partial x_{2}}+\frac{\partial^{2} v_{2}}{\partial x_{1}^{2}}\right|^{2}+\frac{1}{2}\left|\frac{\partial^{2} v_{2}}{\partial x_{1} \partial x_{2}}+\frac{\partial^{2} v_{1}}{\partial x_{2}^{2}}\right|^{2}
\end{aligned}
$$

By

$$
a^{2}+\frac{1}{2}(a+b)^{2} \geq\left(1-\frac{1}{\sqrt{2}}\right)\left(a^{2}+b^{2}\right), \quad a, b \in \mathbb{R} .
$$

We conclude that

$$
\begin{aligned}
& \left|\frac{\partial^{2} v_{1}}{\partial x_{1} \partial x_{2}}\right|^{2}+\frac{1}{2}\left|\frac{\partial^{2} v_{1}}{\partial x_{1} \partial x_{2}}+\frac{\partial^{2} v_{2}}{\partial x_{1}^{2}}\right|^{2} \geq\left(1-\frac{1}{\sqrt{2}}\right)\left(\left|\frac{\partial^{2} v_{1}}{\partial x_{1} \partial x_{2}}\right|^{2}+\left|\frac{\partial^{2} v_{2}}{\partial x_{1}^{2}}\right|^{2}\right) \\
& \left|\frac{\partial^{2} v_{2}}{\partial x_{1} \partial x_{2}}\right|^{2}+\frac{1}{2}\left|\frac{\partial^{2} v_{2}}{\partial x_{1} \partial x_{2}}+\frac{\partial^{2} v_{1}}{\partial x_{2}^{2}}\right|^{2} \geq\left(1-\frac{1}{\sqrt{2}}\right)\left(\left|\frac{\partial^{2} v_{2}}{\partial x_{1} \partial x_{2}}\right|^{2}+\left|\frac{\partial^{2} v_{1}}{\partial x_{2}^{2}}\right|^{2}\right) .
\end{aligned}
$$

Combining the above inequalities, we obtain

$$
|\nabla \epsilon(v)|^{2} \geq(1-1 / \sqrt{2})\left|\nabla^{2} v\right|^{2},
$$

which immediately implies

$$
\left\|\nabla_{h} \epsilon(v)\right\|_{L^{2}}^{2} \geq(1-1 / \sqrt{2})\left\|\nabla_{h}^{2} v\right\|_{L^{2}}^{2}
$$

Combining the above inequalities, we obtain (3.18).

We are ready to prove the error estimate of the proposed elements.

Theorem 3.1. There exists $C$ such that

$$
\begin{aligned}
& \|\left.\left|u-u_{h}\right|\right|_{\iota, h} \leq C h^{r-1}\left(h|u|_{H^{r+1}}+\iota|u|_{H^{r+2}}\right), \quad r \geq 2, \\
& \left\|\left.\left|u-u_{h}\right|\right|_{\iota, h} \leq C h^{1 / 2}\right\| f \|_{L^{2}} .
\end{aligned}
$$

Proof. By the theorem of Berger, Scott and Strang [5], we have, there exists $C$ depends on $\lambda$ and $\mu$ such that

$$
\left\|u-u_{h} \mid\right\|_{\iota, h} \leq C\left(\inf _{v \in V_{h}^{0}}\left|\|u-v \mid\|_{\iota, h}+\sup _{w \in V_{h}^{0}} \frac{E_{h}(u, w)}{\|w \mid\|_{\iota, h}}\right),\right.
$$

where $E_{h}(u, w)=a_{h}(u, w)-\langle f, w\rangle$. 
By [21, Theorem 4], we rewrite the consistency functional $E_{h}$ as

$$
E_{h}(u, w)=\sum_{e \in \mathcal{E}_{h}} \int_{e} n_{i} n_{j} \tau_{i j k}\left[\left[\partial_{n} w_{k}\right]\right] \mathrm{d} t
$$

where $\tau_{i j k}=\iota^{2} \sigma_{j k, i}$ with stress $\sigma=\mathbb{C} \epsilon(u)$. For any $w \in V_{h}^{0}$, we have, for any $e \in \mathcal{E}_{h}$,

$$
\int_{e}\left[\left[\partial_{n} w_{k}\right]\right] \mathrm{d} t=0
$$

We may rewrite $E_{h}$ as

$$
E_{h}(u, w)=\sum_{e \in \mathcal{E}_{h}} \int_{e} n_{i} n_{j}\left(\tau_{i j k}-\Pi_{T} \tau_{i j k}\right)\left[\left[\partial_{n} w_{k}\right]\right] \mathrm{d} t .
$$

Using (3.16a) and the trace inequality (3.13), we obtain

$$
\left|E_{h}(u, w)\right| \leq C h^{r-1} \iota^{2}|u|_{H^{r+2}}\left\|\nabla_{h}^{2} w\right\|_{L^{2}} \leq C h^{r-1} \iota|u|_{H^{r+2}}|||w| \|_{\iota, h} .
$$

Substituting the above inequality, the interpolation estimates (3.16a) into the right-hand side of (3.22), we obtain the desired error estimate (3.21a).

Next, by the trace inequality (3.13) and using the regularity estimate (2.6), we obtain

$$
\left|E_{h}(u, w)\right| \leq C h^{1 / 2} \iota^{2}\left\|\nabla^{2} u\right\|_{L^{2}}^{1 / 2}\left\|\nabla^{2} u\right\|_{H^{1}}^{1 / 2}\left\|\nabla_{h}^{2} w\right\|_{L^{2}} \leq C h^{1 / 2}\|f\|_{L^{2}}\|w\|_{l, h} .
$$

Substituting the above inequality, the interpolation estimates (3.16b) into the right-hand side of (3.22), we obtain the desired error estimate (3.21b).

\section{Numerical examples}

In this part, we test the performance of the proposed elements with $r=2,3$ to resolve solutions with/without boundary layer. We also test the extended rectangular Morley element in [29] for comparison, which is continuous and contains 12 local degrees of freedom. We report the relative errors $\left.||\left|u-u_{h}\right|\left\|_{\iota, h} /\right\||u|\right|_{\iota, h}$ and the rates of convergence on the uniform rectangular mesh with different mesh aspect ratio. In all the tests, we let $\Omega=[0,1]^{2}$ and $\lambda=\mu=1$.

\subsection{First example}

Let $u=\left(u_{1}, u_{2}\right)$ with

$$
u_{1}=\frac{1}{4}(\cos 2 \pi x-1)(\cos 4 \pi y-1), \quad u_{2}=\frac{1}{4}(\cos 4 \pi x-1)(\cos 2 \pi y-1) .
$$

It is clear that $u$ is smooth and there is no boundary layer. The force $f$ is computed by (2.1). We report the relative errors $\left.\left\|\left|u-u_{h}\right|\right\|\right|_{\iota, h} /\|u\| \|_{\iota, h}$ and rates of convergence in 
Table 1: Results of the 1st example with $h=h_{x}=h_{y}$.

\begin{tabular}{|c|c|c|c|c|c|}
\hline$\iota \backslash h$ & $1 / 4$ & $1 / 8$ & $1 / 16$ & $1 / 32$ & $1 / 64$ \\
\hline \multicolumn{6}{|c|}{ Element with $r=2$} \\
\hline $1 \mathrm{e}+0$ & $7.39 \mathrm{e}-01$ & $3.01 \mathrm{e}-01$ & $1.52 \mathrm{e}-01$ & $7.61 \mathrm{e}-02$ & $3.81 \mathrm{e}-02$ \\
\hline rate & & 1.30 & 0.99 & 0.99 & 1.00 \\
\hline $1 \mathrm{e}-2$ & $3.28 \mathrm{e}-01$ & 7.16e-02 & $2.28 \mathrm{e}-02$ & $9.56 \mathrm{e}-03$ & $4.53 e-03$ \\
\hline rate & & 2.19 & 1.65 & 1.26 & 1.08 \\
\hline $1 e-4$ & $3.05 e-01$ & $6.01 \mathrm{e}-02$ & $1.39 \mathrm{e}-02$ & $3.40 \mathrm{e}-03$ & $8.44 \mathrm{e}-04$ \\
\hline rate & & 2.35 & 2.11 & 2.04 & 2.01 \\
\hline $1 e-6$ & $3.06 \mathrm{e}-01$ & $6.01 \mathrm{e}-02$ & $1.39 \mathrm{e}-02$ & $3.39 \mathrm{e}-03$ & $8.49 \mathrm{e}-04$ \\
\hline rate & & 2.35 & 2.11 & 2.04 & 2.01 \\
\hline \multicolumn{6}{|c|}{ Element with $r=3$} \\
\hline $1 e+0$ & $1.10 \mathrm{e}-01$ & $1.09 \mathrm{e}-01$ & $2.56 \mathrm{e}-02$ & $5.62 \mathrm{e}-03$ & $1.28 \mathrm{e}-03$ \\
\hline rate & & 0.02 & 2.09 & 2.19 & 2.13 \\
\hline $1 \mathrm{e}-2$ & $3.15 \mathrm{e}-02$ & $2.31 \mathrm{e}-02$ & $3.67 \mathrm{e}-03$ & $6.97 \mathrm{e}-04$ & $1.52 \mathrm{e}-04$ \\
\hline rate & & 0.45 & 2.65 & 2.40 & 2.20 \\
\hline $1 e-4$ & 2.87e-02 & $1.85 \mathrm{e}-02$ & $1.91 \mathrm{e}-03$ & $2.01 \mathrm{e}-04$ & $2.25 e-05$ \\
\hline rate & & 0.63 & 3.27 & 3.25 & 3.16 \\
\hline $1 e-6$ & $2.87 \mathrm{e}-02$ & $1.85 \mathrm{e}-02$ & $1.91 \mathrm{e}-03$ & $2.00 \mathrm{e}-04$ & $2.24 \mathrm{e}-05$ \\
\hline rate & & 0.63 & 3.27 & 3.25 & 3.16 \\
\hline \multicolumn{6}{|c|}{ Extended Rectangular Morley Element [29] } \\
\hline $1 e+0$ & $6.36 \mathrm{e}-01$ & $2.66 \mathrm{e}-01$ & $1.34 \mathrm{e}-01$ & $6.74 \mathrm{e}-02$ & $3.38 \mathrm{e}-02$ \\
\hline rate & & 1.26 & 0.98 & 1.00 & 1.00 \\
\hline $1 e-2$ & $2.51 \mathrm{e}-01$ & $5.85 \mathrm{e}-02$ & $2.01 \mathrm{e}-02$ & $8.47 \mathrm{e}-03$ & $4.02 \mathrm{e}-03$ \\
\hline rate & & 2.10 & 1.54 & 1.25 & 1.08 \\
\hline $1 \mathrm{e}-4$ & $2.34 \mathrm{e}-01$ & $4.75 \mathrm{e}-02$ & $1.22 \mathrm{e}-02$ & $3.08 \mathrm{e}-3$ & $7.72 \mathrm{e}-04$ \\
\hline rate & & 2.30 & 1.96 & 1.99 & 1.99 \\
\hline $1 e-6$ & $2.34 \mathrm{e}-01$ & $4.75 \mathrm{e}-02$ & $1.22 \mathrm{e}-02$ & $3.08 \mathrm{e}-03$ & 7.71-04 \\
\hline rate & & 2.30 & 1.96 & 1.99 & 2.00 \\
\hline
\end{tabular}

Table 1 for uniform mesh with $h_{x}=h_{y}$ and the same quantities are shown in Table 2 for mesh with $h_{x}=2 h_{y}$.

In view of the above results, for $r=2,3$, we observe $(r-1)$-order convergence when $\iota=1$ and $r$-order convergence when $\iota$ approaches zero for the proposed element, which is consistent with Theorem 3.1. Compared with the extended rectangular Morley element, our element with $r=2$ already can ensure same order convergence with the same number of degrees of freedom.

\subsection{Second example}

In this part, we test the proposed elements with degree $r=2$ and $r=3$ to resolve a solution with strong boundary layer effect. We also list the results for the extended high order 
Table 2: Results of the 1st example with $h=h_{x}=2 h_{y}$.

\begin{tabular}{|c|c|c|c|c|c|}
\hline$\iota \backslash h$ & $1 / 4$ & $1 / 8$ & $1 / 16$ & $1 / 32$ & $1 / 64$ \\
\hline \multicolumn{6}{|c|}{ Element with $r=2$} \\
\hline $1 \mathrm{e}+0$ & $6.80 \mathrm{e}-01$ & $2.69 \mathrm{e}-01$ & $1.35 \mathrm{e}-01$ & $6.75 \mathrm{e}-02$ & $3.37 \mathrm{e}-02$ \\
\hline rate & & 1.34 & 1.00 & 1.00 & 1.00 \\
\hline $1 e-2$ & $3.89 \mathrm{e}-01$ & $6.79 \mathrm{e}-02$ & $2.04 \mathrm{e}-02$ & $8.47 \mathrm{e}-03$ & $4.01 \mathrm{e}-03$ \\
\hline rate & & 2.52 & 1.73 & 1.27 & 1.08 \\
\hline $1 e-4$ & 3.37e-01 & $5.56 \mathrm{e}-02$ & $1.22 \mathrm{e}-02$ & $2.92 \mathrm{e}-03$ & $7.24 \mathrm{e}-04$ \\
\hline rate & & 2.60 & 2.19 & 2.06 & 2.01 \\
\hline $1 e-6$ & 3.37e-01 & $5.56 \mathrm{e}-02$ & $1.22 \mathrm{e}-02$ & $2.92 \mathrm{e}-03$ & $7.23 \mathrm{e}-04$ \\
\hline rate & & 2.60 & 2.19 & 2.06 & 2.01 \\
\hline \multicolumn{6}{|c|}{ Element with $r=3$} \\
\hline $1 \mathrm{e}+0$ & $1.51 \mathrm{e}-01$ & $7.39 \mathrm{e}-02$ & $1.64 \mathrm{e}-02$ & $3.79 \mathrm{e}-03$ & $9.07 e-04$ \\
\hline rate & & 1.03 & 2.17 & 2.11 & 2.06 \\
\hline $1 \mathrm{e}-2$ & $4.37 \mathrm{e}-02$ & $1.30 \mathrm{e}-02$ & $2.31 \mathrm{e}-03$ & $4.71 \mathrm{e}-04$ & $1.08 \mathrm{e}-04$ \\
\hline rate & & 1.75 & 2.49 & 2.29 & 2.13 \\
\hline $1 \mathrm{e}-4$ & $3.89 \mathrm{e}-02$ & 8.87e-03 & $1.04 \mathrm{e}-03$ & $1.25 \mathrm{e}-04$ & $1.53 \mathrm{e}-05$ \\
\hline rate & & 2.13 & 3.09 & 3.06 & 3.02 \\
\hline $1 e-6$ & $3.89 \mathrm{e}-02$ & 8.87e-03 & $1.04 \mathrm{e}-03$ & $1.25 \mathrm{e}-04$ & $1.53 \mathrm{e}-05$ \\
\hline rate & & 2.13 & 3.09 & 3.07 & 3.03 \\
\hline \multicolumn{6}{|c|}{ Extended Rectangular Morley Element [29] } \\
\hline $1 \mathrm{e}+0$ & $6.12 \mathrm{e}-01$ & $2.53 \mathrm{e}-01$ & $1.28 \mathrm{e}-01$ & $6.43 e-02$ & $3.22 \mathrm{e}-02$ \\
\hline rate & & 1.27 & 0.98 & 1.00 & 1.00 \\
\hline $1 \mathrm{e}-2$ & $2.85 \mathrm{e}-01$ & $5.80 \mathrm{e}-02$ & $1.91 \mathrm{e}-02$ & $8.04 \mathrm{e}-03$ & $3.83 e-03$ \\
\hline rate & & 2.29 & 1.60 & 1.25 & 1.07 \\
\hline $1 e-4$ & $2.57 \mathrm{e}-01$ & $4.51 \mathrm{e}-02$ & $1.10 \mathrm{e}-02$ & 2.77e-03 & $6.93 \mathrm{e}-04$ \\
\hline rate & & 2.51 & 2.03 & 2.00 & 2.00 \\
\hline $1 e-6$ & $2.57 \mathrm{e}-01$ & $4.51 \mathrm{e}-02$ & $1.10 \mathrm{e}-02$ & 2.77e-03 & $6.92 \mathrm{e}-04$ \\
\hline rate & & 2.51 & 2.03 & 2.00 & 2.00 \\
\hline
\end{tabular}

rectangular Morley element for comparison. The solution $u=\left(u_{1}, u_{2}\right)$ is given by

$$
\begin{aligned}
& u_{1}=\left(\exp (\sin \pi x)-1-\pi \iota \frac{1+\exp (-1 / \iota)-\exp [(x-1) / \iota]-\exp (-x / \iota)}{1-\exp (-1 / \iota)}\right) \\
& \times\left(\exp (\sin \pi y)-1-\pi \iota \frac{1+\exp (-1 / \iota)-\exp [(x-1) / \iota]-\exp (-x / \iota)}{1-\exp (-1 / \iota)}\right), \\
& u_{2}=\left(\sin \pi x-\pi \iota \frac{1+\exp (-1 / \iota)-\exp [(x-1) / \iota]-\exp (-x / \iota)}{1-\exp (-1 / \iota)}\right) \\
& \quad \times\left(\sin \pi x-\pi \iota \frac{1+\exp (-1 / \iota)-\exp [(x-1) / \iota]-\exp (-x / \iota)}{1-\exp (-1 / \iota)}\right) .
\end{aligned}
$$

We plot $\partial u_{1} / \partial x$ with $\iota=1 e-6$ in the left sub-figure of Fig. 1 , which shows that $\nabla u$ has a layer. The cross section with $y=1 / 2$ of $\partial u_{1} / \partial x$ is drawn in the right sub-figure.

The force $f$ is also computed by (2.1). For the solution with boundary layer effect, the results of relative errors $\left|\left\|u-u_{h} \mid\right\|_{\iota, h} /\|\| u \|_{\iota, h}\right.$ and the rates of convergence on mesh 

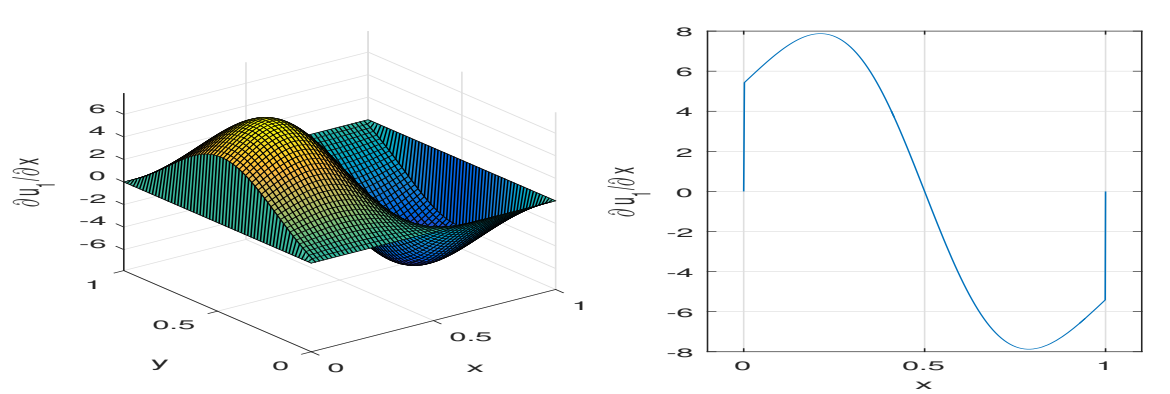

Figure 1: Plot of $\partial u_{1} / \partial x$ with $\iota=1 e-6$ in $\Omega$, Left: plot over $\Omega=[0,1]^{2}$; Right: plot over the cross section $[0,1] \times\{1 / 2\}$.

with $h_{x}=h_{y}$ are shown in Table 3 and on mesh with $h_{x}=2 h_{y}$ are shown in Table 4 . It follows from these tables that the rate of convergence for the proposed element with $r=2,3$ changes from $r-1$ to $1 / 2$ as $\iota \rightarrow 0$. The same scenario is observed for the extended rectangular Morley element.

\section{Appendix}

\section{A Element with $r=2$}

We list the basis functions for the elements with $r=2,3$, which are constructed by the method described in Section 3. Denote by $\left\{\left(x_{i}, y_{i}\right)\right\}_{i=1}^{4}$ the coordinates for the vertices of $K$ from lower left to the upper left in a counterclockwise way.

In this part, we describe the element with $r=2$. The element $\left(K, P_{K}, \Sigma_{K}\right)$ is defined as follows.

1. $P_{K}:=\mathcal{S}_{2}(K)+\operatorname{span}\left\{b_{K} b_{e_{i}} \mid, i=1, \cdots, 4\right\}$. Here

$$
\mathcal{S}_{2}(K)=\operatorname{span}\left\{1, x, y, x^{2}, x y, y^{2}, x^{2} y, x y^{2}\right\}
$$

2. For all $v \in P_{K}$, the set of degrees of freedom

$$
\Sigma_{K}:=\left\{v\left(a_{i}\right), \int_{e_{i}} v \mathrm{~d} s, \int_{e_{i}} \frac{\partial v}{\partial n} \mathrm{~d} s \mid i=1, \cdots, 4\right\} .
$$

Let $\left\{\psi_{i}\right\}_{i=1}^{12}$ be the basis functions of $P_{K}$ : The first four basis functions associated with four vertices are

$$
\psi_{i}=\frac{1}{8}\left(1+x_{i} x\right)\left(1+y_{i} y\right)\left(3 x_{i} x+3 y_{i} y-4\right)-\frac{3}{2}\left(\psi_{9}+\psi_{10}-\psi_{11}-\psi_{12}\right), \quad i=1, \cdots, 4 .
$$


Table 3: Results of the 2nd example with $h=h_{x}=h_{y}$.

\begin{tabular}{|c|c|c|c|c|c|}
\hline$\iota \backslash h$ & $1 / 4$ & $1 / 8$ & $1 / 16$ & $1 / 32$ & $1 / 64$ \\
\hline \multicolumn{6}{|c|}{ Element with $r=2$} \\
\hline $1 \mathrm{e}+0$ & $2.99 \mathrm{e}-01$ & $1.41 \mathrm{e}-01$ & $7.03 e-02$ & $3.52 \mathrm{e}-02$ & $1.76 \mathrm{e}-02$ \\
\hline rate & & 1.08 & 1.01 & 1.00 & 1.00 \\
\hline $1 e-2$ & $2.06 \mathrm{e}-01$ & $1.30 \mathrm{e}-01$ & 8.33e-02 & $5.19 \mathrm{e}-02$ & $2.91 \mathrm{e}-02$ \\
\hline rate & & 0.67 & 0.64 & 0.68 & 0.83 \\
\hline $1 e-4$ & $2.40 \mathrm{e}-01$ & $1.64 \mathrm{e}-01$ & $1.15 \mathrm{e}-01$ & $8.13 \mathrm{e}-02$ & $5.73 e-02$ \\
\hline rate & & 0.55 & 0.51 & 0.50 & 0.51 \\
\hline $1 e-6$ & $2.40 \mathrm{e}-01$ & $1.64 \mathrm{e}-01$ & $1.15 \mathrm{e}-01$ & $8.13 \mathrm{e}-02$ & $5.74 \mathrm{e}-02$ \\
\hline rate & & 0.55 & 0.51 & 0.50 & 0.50 \\
\hline \multicolumn{6}{|c|}{ Element with $r=3$} \\
\hline $1 \mathrm{e}+0$ & $1.91 \mathrm{e}-01$ & $5.34 \mathrm{e}-02$ & $1.15 \mathrm{e}-02$ & $2.47 \mathrm{e}-03$ & $5.58 \mathrm{e}-04$ \\
\hline rate & & 1.84 & 2.21 & 2.22 & 2.14 \\
\hline $1 \mathrm{e}-2$ & $1.29 \mathrm{e}-01$ & $7.49 \mathrm{e}-02$ & $3.81 \mathrm{e}-02$ & $1.57 \mathrm{e}-02$ & $5.00 \mathrm{e}-03$ \\
\hline rate & & 0.78 & 0.98 & 1.28 & 1.65 \\
\hline $1 \mathrm{e}-4$ & $1.77 \mathrm{e}-01$ & $1.24 \mathrm{e}-01$ & 8.70e-02 & $6.13 e-02$ & $4.31 \mathrm{e}-02$ \\
\hline rate & & 0.52 & 0.51 & 0.50 & 0.51 \\
\hline $1 e-6$ & $1.77 \mathrm{e}-01$ & $1.24 \mathrm{e}-01$ & $8.69 \mathrm{e}-02$ & $6.13 e-02$ & $4.33 \mathrm{e}-02$ \\
\hline rate & & 0.52 & 0.51 & 0.50 & 0.50 \\
\hline \multicolumn{6}{|c|}{ Extended Rectangular Morley Element [29] } \\
\hline $1 \mathrm{e}+0$ & $2.48 \mathrm{e}-01$ & $1.23 \mathrm{e}-01$ & $6.14 \mathrm{e}-02$ & $3.07 e-02$ & $1.54 \mathrm{e}-02$ \\
\hline rate & & 1.01 & 1.00 & 1.00 & 1.00 \\
\hline $1 \mathrm{e}-2$ & $1.83 \mathrm{e}-01$ & $1.14 \mathrm{e}-01$ & $7.10 \mathrm{e}-02$ & $4.39 \mathrm{e}-02$ & $2.48 \mathrm{e}-02$ \\
\hline rate & & 0.68 & 0.69 & 0.69 & 0.82 \\
\hline $1 e-4$ & $2.20 \mathrm{e}-01$ & $1.54 \mathrm{e}-01$ & $1.08 \mathrm{e}-01$ & 7.63e-02 & $5.38 \mathrm{e}-02$ \\
\hline rate & & 0.52 & 0.51 & 0.50 & 0.51 \\
\hline $1 e-6$ & $2.20 \mathrm{e}-01$ & $1.54 \mathrm{e}-01$ & $1.08 \mathrm{e}-01$ & $7.63 e-02$ & $5.39 \mathrm{e}-02$ \\
\hline rate & & 0.52 & 0.51 & 0.50 & 0.50 \\
\hline
\end{tabular}

Four basis functions associated with $\left\{\int_{e_{i-4}} v\right\}_{i=5}^{8}$ are

$$
\begin{aligned}
& \psi_{i}=\frac{3}{8}\left(1-x^{2}\right)\left(1+y_{i-4} y\right)+\frac{1}{2} y_{i-4}\left(\psi_{9}+\psi_{11}\right)+\frac{3}{2}\left(\psi_{10}-\psi_{12}\right), \quad i=5,7, \\
& \psi_{i}=\frac{3}{8}\left(1-y^{2}\right)\left(1+x_{i-4} x\right)+\frac{3}{2}\left(\psi_{9}-\psi_{11}\right)-\frac{1}{2} x_{i-4}\left(\psi_{10}+\psi_{12}\right), \quad i=6,8 .
\end{aligned}
$$

The last four basis functions are

$$
\psi_{9}=-30 b_{K} b_{e_{1}}, \quad \psi_{10}=-30 b_{K} b_{e_{2}}, \quad \psi_{11}=30 b_{K} b_{e_{3}}, \quad \psi_{12}=30 b_{K} b_{e_{4}} .
$$

\section{B Element with $r=3$}

The element $\left(K, P_{K}, \Sigma_{K}\right)$ is defined as follows 
Table 4: Results of the 2nd example with $h=h_{x}=2 h_{y}$.

\begin{tabular}{|c|c|c|c|c|c|}
\hline$\iota \backslash h$ & $1 / 4$ & $1 / 8$ & $1 / 16$ & $1 / 32$ & $1 / 64$ \\
\hline \multicolumn{6}{|c|}{ Element with $r=2$} \\
\hline $1 \mathrm{e}+0$ & $3.08 \mathrm{e}-01$ & $1.53 \mathrm{e}-01$ & $7.64 \mathrm{e}-02$ & $3.82 \mathrm{e}-02$ & $1.91 \mathrm{e}-02$ \\
\hline rate & & 1.01 & 1.00 & 1.00 & 1.00 \\
\hline $1 \mathrm{e}-2$ & $2.04 \mathrm{e}-01$ & $1.32 \mathrm{e}-01$ & $8.91 \mathrm{e}-02$ & $5.47 \mathrm{e}-02$ & $2.99 \mathrm{e}-02$ \\
\hline rate & & 0.62 & 0.57 & 0.70 & 0.87 \\
\hline $1 \mathrm{e}-4$ & $2.29 \mathrm{e}-01$ & $1.54 \mathrm{e}-01$ & $1.08 \mathrm{e}-01$ & $7.62 \mathrm{e}-02$ & $5.33 e-02$ \\
\hline rate & & 0.57 & 0.51 & 0.51 & 0.52 \\
\hline $1 e-6$ & $2.29 \mathrm{e}-01$ & $1.54 \mathrm{e}-01$ & $1.08 \mathrm{e}-01$ & $7.62 \mathrm{e}-02$ & $5.38 \mathrm{e}-02$ \\
\hline rate & & 0.57 & 0.51 & 0.50 & 0.50 \\
\hline \multicolumn{6}{|c|}{ Element with $r=3$} \\
\hline $1 e+0$ & $1.28 \mathrm{e}-01$ & $2.94 \mathrm{e}-02$ & $6.28 \mathrm{e}-03$ & $1.41 \mathrm{e}-03$ & $3.33 \mathrm{e}-04$ \\
\hline rate & & 2.21 & 2.22 & 2.16 & 2.08 \\
\hline $1 \mathrm{e}-2$ & $1.18 \mathrm{e}-01$ & 7.07e-02 & $3.66 \mathrm{e}-02$ & $1.50 \mathrm{e}-02$ & $4.75 e-03$ \\
\hline rate & & 0.74 & 0.95 & 1.29 & 1.66 \\
\hline $1 \mathrm{e}-4$ & $1.63 \mathrm{e}-01$ & $1.14 \mathrm{e}-01$ & $8.01 \mathrm{e}-02$ & $5.65 e-02$ & $3.93 e-02$ \\
\hline rate & & 0.51 & 0.51 & 0.50 & 0.52 \\
\hline $1 e-6$ & $1.63 \mathrm{e}-01$ & $1.14 \mathrm{e}-01$ & $8.01 \mathrm{e}-02$ & $5.65 e-02$ & $3.99 \mathrm{e}-02$ \\
\hline rate & & 0.51 & 0.51 & 0.50 & 0.50 \\
\hline \multicolumn{6}{|c|}{ Extended Rectangular Morley Element [29] } \\
\hline $1 \mathrm{e}+0$ & $2.71 \mathrm{e}-01$ & $1.37 \mathrm{e}-01$ & $6.87 \mathrm{e}-02$ & $3.44 \mathrm{e}-02$ & $1.72 \mathrm{e}-02$ \\
\hline rate & & 0.99 & 0.99 & 1.00 & 1.00 \\
\hline $1 \mathrm{e}-2$ & $1.63 \mathrm{e}-01$ & $1.04 \mathrm{e}-01$ & $7.08 \mathrm{e}-02$ & $4.62 \mathrm{e}-02$ & $2.62 \mathrm{e}-02$ \\
\hline rate & & 0.65 & 0.55 & 0.62 & 0.82 \\
\hline $1 \mathrm{e}-4$ & $1.977 \mathrm{e}-01$ & $1.37 \mathrm{e}-01$ & $9.62 \mathrm{e}-02$ & $6.79 \mathrm{e}-02$ & $4.74 \mathrm{e}-02$ \\
\hline rate & & 0.53 & 0.51 & 0.50 & 0.52 \\
\hline $1 e-6$ & $1.98 \mathrm{e}-01$ & $1.37 \mathrm{e}-01$ & $9.62 \mathrm{e}-02$ & $6.79 \mathrm{e}-02$ & $4.80 \mathrm{e}-02$ \\
\hline rate & & 0.53 & 0.51 & 0.50 & 0.50 \\
\hline
\end{tabular}

1. $P_{K}:=\mathcal{S}_{3}(K)+b_{K} \sum_{i=1}^{4} b_{e_{i}} \Lambda_{e_{i}}$. Here $\Lambda_{e_{i}}$ are shown in (3.6) and

$$
\mathcal{S}_{3}(K)=\operatorname{span}\left\{1, x, y, x^{2}, x y, y^{2}, x^{3}, x^{2} y, x y^{2}, y^{3}, x^{3} y, x y^{3}\right\}
$$

2. For all $v \in P_{K}$, the set of degrees of freedom

$$
\begin{aligned}
\Sigma_{K}:=\left\{v\left(a_{i}\right), \int_{e_{i}} v \mathrm{~d} s, \int_{e_{i}} \frac{\partial v}{\partial n} \mathrm{~d} s \mid i=1, \cdots, 4\right\} \\
\cup\left\{\int_{e_{i}} P_{1}^{(2,2)}(x) v \mathrm{~d} s, \int_{e_{i}} P_{1}^{(2,2)}(x) \frac{\partial v}{\partial n} \mathrm{~d} s \mid i=1,3\right\} \\
\cup\left\{\int_{e_{i}} P_{1}^{(2,2)}(y) v \mathrm{~d} s, \int_{e_{i}} P_{1}^{(2,2)}(y) \frac{\partial v}{\partial n} \mathrm{~d} s \mid i=2,4\right\} .
\end{aligned}
$$


Let $\left\{\psi_{i}\right\}_{i=1}^{20}$ be the basis functions of $P_{K}$ : The first four basis functions associated with four vertices are

$$
\begin{aligned}
\psi_{i}= & \frac{1}{8} \\
& \left(1+x_{i} x\right)\left(1+y_{i} y\right)\left(5 x^{2}+5 y^{2}-2 x_{i} x-2 y_{i} y-4\right) \\
& -x_{i}\left(\psi_{i \bmod 4+13}+4 \psi_{(i+2) \bmod 4+13}\right)+y_{i}\left(4 \psi_{i+12}+\psi_{(i+1) \bmod 4+13}\right) \\
& +4\left(\psi_{i+16}-\psi_{(i+2) \bmod 4+17}\right)-\left(\psi_{i \bmod 4+17}-\psi_{(i+1) \bmod 4+17}\right), \quad i=1, \cdots, 4 .
\end{aligned}
$$

Four basis functions associated with $\left\{\int_{e_{i-4}} v\right\}_{i=5}^{8}$ are: for $i=5,7$,

$$
\psi_{i}=\frac{3}{8}\left(1-x^{2}\right)\left(1+y_{i-4} y\right)+\frac{1}{2} y_{i-4}\left(\psi_{13}+\psi_{15}\right)+\frac{3}{2}\left(\psi_{14}-\psi_{16}\right)+\frac{3}{2} y_{i-4}\left(\psi_{18}-\psi_{20}\right),
$$

and for $i=6,8$,

$$
\psi_{i}=\frac{3}{8}\left(1-y^{2}\right)\left(1+x_{i-4} x\right)+\frac{3}{2}\left(\psi_{13}-\psi_{15}\right)-\frac{1}{2} x_{i-4}\left(\psi_{14}+\psi_{16}\right)+\frac{3}{2} x_{i-4}\left(\psi_{17}-\psi_{19}\right) .
$$

Four basis functions associated with $\left\{\int_{e_{i-8}} P_{1}^{(2,2)} v\right\}_{i=9}^{12}$ are

$$
\begin{aligned}
\psi_{i}= & \frac{5}{8} x\left(1-x^{2}\right)\left(1+y_{i-8} y\right)+\frac{5}{2}\left(\psi_{14}+\psi_{16}\right)+\frac{1}{2} y_{i-8}\left(\psi_{17}+\psi_{19}\right) \\
& +\frac{5}{2} y_{i-8}\left(\psi_{18}+\psi_{20}\right), \quad i=9,11, \\
\psi_{i}= & \frac{5}{8} y\left(1-y^{2}\right)\left(1+x_{i-8} x\right)-\frac{5}{2}\left(\psi_{13}+\psi_{15}\right)-\frac{5}{2} x_{i-8}\left(\psi_{17}+\psi_{19}\right) \\
& \quad-\frac{1}{2} x_{i-8}\left(\psi_{18}+\psi_{20}\right), \quad i=10,12 .
\end{aligned}
$$

Four basis functions associated with $\left\{\int_{e_{-12} i} \partial v / \partial n\right\}_{i=13}^{16}$ are

$$
\begin{array}{ll}
\psi_{13}=15 b_{K} b_{e_{1}} P_{1}^{(2,1)}(y), & \psi_{14}=-15 b_{K} b_{e_{2}} P_{1}^{(1,2)}(x), \\
\psi_{15}=15 b_{K} b_{e_{2}} P_{1}^{(1,2)}(y), & \psi_{16}=-15 b_{K} b_{e_{1}} P_{1}^{(2,1)}(x) .
\end{array}
$$

Four basis functions associated with $\left\{\int_{e_{i-16}} P_{1}^{(2,2)} \partial v / \partial n\right\}_{i=17}^{20}$ are

$$
\begin{array}{ll}
\psi_{17}=-\frac{70}{3} b_{K} b_{e_{1}} P_{1}^{(2,2)}(x), & \psi_{18}=-\frac{70}{3} b_{K} b_{e_{2}} P_{1}^{(2,2)}(y), \\
\psi_{19}=\frac{70}{3} b_{K} b_{e_{3}} P_{1}^{(2,2)}(x), & \psi_{20}=\frac{70}{3} b_{K} b_{e_{4}} P_{1}^{(2,2)}(y) .
\end{array}
$$

\section{Acknowledgements}

The work of Ming was partially supported by the National Natural Science Foundation of China for Distinguished Young Scholars No. 11425106 and National Natural Science Foundation of China grants No. 91630313 and by the support of CAS NCMIS. 


\section{References}

[1] R. A. Adams And J. J. F. Fournier, Sobolev Spaces, Academic Press, 2nd ed., 2003.

[2] S. B. AltAN AND E. C. AIfANTIS, On the structure of the mode III crack-tip in gradient elasticity, Scripta Metal. Mater., 26 (1992), pp. 319-324.

[3] J. H. ARGYRIS, I. FRIED AND D. W. SCHARPF, The Tuba family of plate elements for the matrix displacement method, The Aeronautical Journal of the Royal Aeronautical Society, 72 (1968), pp. 701-709.

[4] D. N. ARnOld AND G. Awanou, The serendipity family of finite elements, Found. Comput. Math., 11 (2011), pp. 337-344.

[5] A. Berger, L. R. SCOTt AND G. STRANG, Approximate boundary conditions in the finite element method, Symposia Math., X (1972), pp. 295-313.

[6] F. A. BOGNER, R. L. Fox AND L. A. SCHMIT, The generation of interelement compatible stiffness and mass matrices by the use of interpolation formulas, Proceedings of the Conferences on the Matrix Methods in Structural Mechanics, Wright Patterson A. F. B. Ohio, 1965.

[7] D. BRAESS, Finite Element: Theory, Fast Solvers and Applications In Solid Mechanics, Cambridge University Press, Cambridge, 3rd eds., 2007.

[8] S. C. BRENNER, $C^{0}$ interior penalty method, in Fronters in Numerical Analysis-Durham 2010, edited by L. Blowey and M. Jensen, Lecture Notes in Computational Science and Engineering 85, Springer-Verlag Berlin Heidelberg, 2012, pp. 79-147.

[9] S. C. BRENNER AND L.-Y. SUNG, $C^{0}$ interior penalty methods for fourth order elliptic boundary value problems on polygonal domains, J. Sci. Comput., 22/23 (2005), pp. 83-118.

[10] H. R. CHEN AND S. C. CHEN, $C^{0}$-nonconforming elements for fourth order elliptic problem, Math. Numer. Sinica, 35 (2013), pp. 21-30.

[11] P. G. Ciarlet, The Finite Element Method for Elliptic Problems, North-Holland, Amsterdam, 1978.

[12] E. Cosserar And F. Cosserat, Theorie des Corps Deformables, Pair: Herman et fils (1909).

[13] G. E. EXADAKTYLOS AND E. C. AIFANTIS, Two and three dimensional crack problems in gradient elasticity, J. Mech. Behav. Mater., 7 (1996), pp. 93-118.

[14] P. Fisher, J. MERGHeim AND P. STEINMANN, On the $C^{1}$ continuous discretization of nonlinear gradient elasticity: a comparison of NEM and FEM based on Bernstein-Bézier patches, Int. J. Numer. Meth. Eng., 82 (2010), pp. 1282-1307.

[15] N. A. FleCK AND J. W. HutCHINSON, Strain gradient plasticity, Advances in Applied Mechanics, Vol. 33, Academic Press, 1997, pp. 295-361.

[16] M. S. FloAter AND A. Gillette, Nodal bases for the serendipity family of finite elements, Found. Comput. Math., 17 (2017), pp. 879-893.

[17] B. GAO, S. ZHANG AND M. WANG, A note on the nonconforming finite elements for elliptic problem, J. Comput. Math., 29 (2011), pp. 215-226.

[18] P. GRISVARD, Elliptic Problems in Nonsmooth Domains, Pitman, Boston, 1985.

[19] J. GUZMÁN, D. LEYKEKHMAN AND M. NEILAN, A family of non-conforming elements and the analysis of Nitsche's method for a singularly perturbed fourth order problem, Calcolo, 49 (2012), pp. 95-125.

[20] J. HU, Y. Q. HUANG AND S. Y. ZHANG, The lowest order differentiable finite element on rectangular grids, SIAM J. Numer. Anal., 49 (2011), pp. 1350-1368.

[21] H. L. LI, P. B. MING AND Z.-C. SHI, Two robust nonconforming $\mathrm{H}^{2}$-elements for linear strain gradient elasticity, Numer. Math., 137 (2017), pp. 691-711.

[22] H. L. LI, P. B. MING AND Z.-C. SHI, New Nonconforming Elements for Linear Strain 
Gradient Elastic Model, 2018, arXiv:109.00819.

[23] L. S. D. MORLEY, The triangular equilibrium problem in the solution of plate bending problems, Aero. Quart., 19 (1968), pp. 149-169.

[24] S.-A. Papanastasiou, A. Zervos and I. Vardoulakis, A three-dimensional $C^{1}$ finite element for gradient elasticity, Int. J. Numer. Meth. Eng., 135 (2009), pp. 1396-1415.

[25] J. Petera AND J. PitTMAn, Isoparametric Hermite elements, Inter. J. Numer. Meth. Eng., 37 (1994), pp. 1489-1519.

[26] C. Q. RU AND E. C. AIFANTIS, A simple approach to solve boundary-value problems in gradient elasticity, Acta Mech., 101 (1993), pp. 59-68.

[27] J. Y. SHU, W. E. KING AND N. A. FLECK, Finite elements for materials with strain gradient effects, Internat. J. Numer. Meth. Eng., 44 (1999), pp. 373-391.

[28] G. SzEGÖ, Orthnogonal Polynomials, AMS, Providence, Rhode Island, 1975, AMS Coll. Publ. Vol. XXIII, 4th eds.

[29] L. WANG, Y. K. WU AND X. P. XIE, Uniformly stable rectangular elements for fourth order elliptic singular perturbation problems, Numer. Methods Partial Differ. Equations, 29 (2013), pp. 721737.

[30] A. Zervos, S.-A. Papanastasiou AND I. VARDOUlaKis, Two finite element discretizations for gradient elasticity, J. Eng. Mech., 135 (2009), pp. 203-213.

[31] S. Y. ZHANG, On the full $\mathcal{C}^{1}-\mathcal{Q}_{k}$ finite element spaces on rectangles and cuboids, Adv. Appl. Math. Mech., 2 (2010), pp. 701-721. 